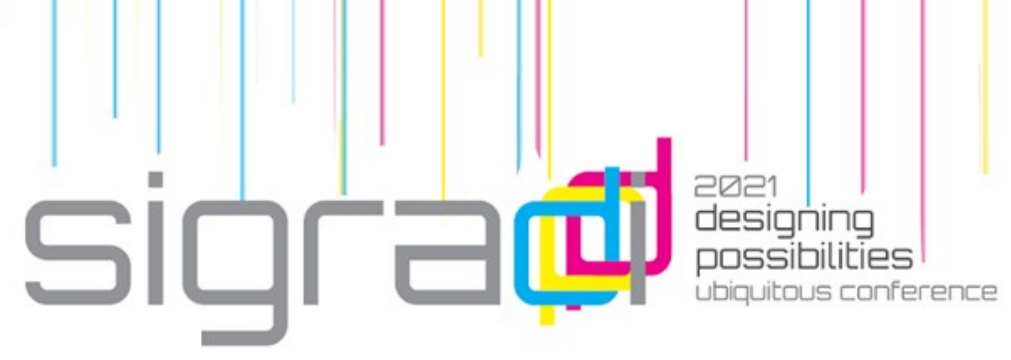

\title{
Comparative Study for Several BIM Software on Latin-American AEC Market
}

Danny Lobos ${ }^{1}$, Katherinne Rojas ${ }^{1}$, Jael Millán ${ }^{1}$, Rodolfo Palma ${ }^{1}$, Pablo Morel ${ }^{1}$, Rodrigo Vallejos ${ }^{1}$, Eber $\mathrm{Cruz}^{1}$, Fernando Olguín ${ }^{1}$, Víctor Núñez ${ }^{1}$

\author{
${ }^{1}$ Universidad Tecnológica Metropolitana, Chile \\ danny.lobosc@utem.cl \\ k.rojas@utem.cl \\ jael.millan@utem.cl \\ rpalmastgo@hotmail.com \\ morel.arq@gmail.com \\ vallejos.tecnica@gmail.com \\ acceso.company@gmail.com \\ folguinm@utem.cl \\ vgnunez@uc.cl
}

\begin{abstract}
There is a variety of BIM brands present in the Latin American market, there are several questions about their performance in the Spanish language. BIM software companies with a presence in Latin America were appointed to a series of structured and sequenced workshop. At the end of each talk, a specialized audience responded to a series of structured surveys on both theoretical and practical aspects of the software presented. The demonstration consisted of two areas: one theoretical and one practical. The theory presented relevant historical attributes, brand visibility data and some known examples of its use. In the practical part, a live demonstration of the use of the basic tools and functions of the interface was requested. It was derived that: The companies fulfilled the expectation on the agenda in a dissimilar way, the responses to the surveys revealed a similar performance in general terms (modeling, prices), the workshops showed significant differences in theoretical aspects such as known examples, certification. There is a need to advance in the dissemination of the performance of the different BIM brands in the AEC industry in Latin America, the level of individual experience strongly influences the evaluation of the software, the user's profile and the type of project will determine the software to use.
\end{abstract}

Keywords: BIM, education, software, workshop, research. 


\section{INTRODUCCIÓN}

Frente al estado actual de las tecnologías BIM en el Chile, varias estadísticas avalan (Loyola, 2019; Loyola, 2016; Loyola, 2013; PlanBIM, 2019; IALE Tecnología, 2017) el crecimiento de su adopción se ha desarrollado el "Estándar BIM para Proyectos Públicos", y la importancia de la exploración desde esfuerzos nacionales por la incorporación de BIM en el diseño y construcción en el país. Frente a la variedad de marcas BIM presentes en el mercado AEC Latinoamericano existen diversas interrogantes sobre el desempeño integral de los softwares ante los requerimientos de los proyectos de construcción en el continente. Normalmente las investigaciones y comparaciones están hechas en el hemisferio norte y en inglés (Jobim et al., 2017; Jones, 2014; McGraw-Hill, 2014) y consideran un desempeño similar a las distintas marcas. Sin embargo, estos estudios no han considerado actualizaciones, complementos ni las versiones más recientes de los softwares, tampoco han contemplado la participación de audiencias especializadas en estas tecnologías. Las empresas de software normalmente ofrecen sus productos a través de diversas estrategias de marketing, como seminarios, demostraciones, licencias de estudiantes, periodos de pruebas, entre otros. El alcance de las distintas casas de softwares con los usuarios se ve fuertemente influenciado por la predominancia de la empresa Autodesk en el mercado chileno.

El estudio realizado por (Khemlani, 2010) revisa todos los software BIM presentes en el mercado americano, y orienta sobre sus desempeños, sin embargo, solo estaba disponible en idioma inglés. Se centró principalmente en evaluar las capacidades técnicas de cada aplicación, los aspectos de implementación, precios, capacitaciones, soporte técnico y posición de mercado y potencial a largo plazo. (Eastman et al., 2011) analiza el desempeño de las marcas para el mercado norteamericano, pero no profundiza en aspectos técnicos ni uso de cada una.

De acuerdo con (Loyola, 2019), que tenía como objetivo caracterizar la utilización de plataformas BIM en el sector privado de la industria Arquitectura, Ingeniería y Construcción en Chile, este arrojó como resultado que la mitad de los encuestados (instrumento de 40 preguntas) utilizaba regularmente herramientas BIM tales como Revit y Navisworks, el resto lo hace ocasionalmente. La limitante de una encuesta online cuya respuesta es optativa para el receptor del correo, conlleva un sesgo natural hacia quienes tienen interés en el tema de la encuesta, lo que se manifiesta en una baja tasa de respuesta y resultados sesgados positivamente (más altos) que los que se 


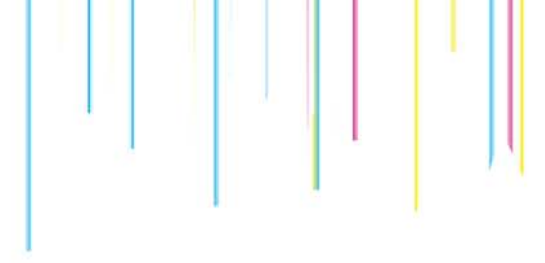

respectivas para estimular y enriquecer la experiencia de la demostración y abrir espacios a consultas nacidas en el momento de la exploración del interfaz del software. A continuación, imágenes de dichas experiencias (Allplan: Figura 3, Vectorworks: Figura 4, Archicad: Figura 5).

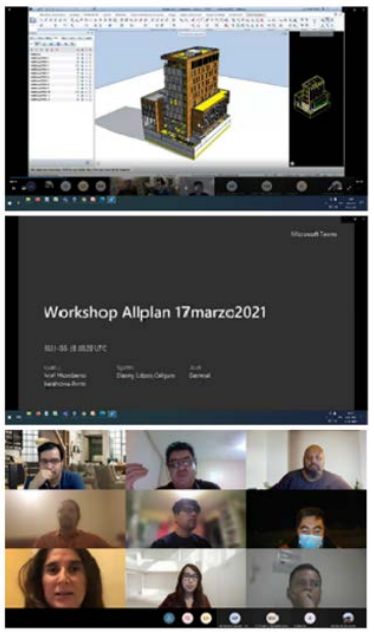

Figura 3 Inicio de Sesión

- Exposición teórica; Ciclo Preguntas y

Respuestas;

Demostración de Interfaz del Software. Fuente: Elaboración propia + Sebastián González, 2021

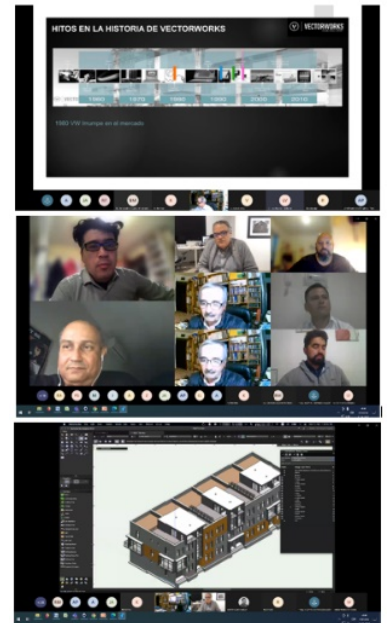

Figura 4 Inicio de Sesión - Exposición teórica; Ciclo Preguntas y Respuestas; Demostración de Interfaz del Software. Fuente:

Elaboración propia +

Rubén Hernández, 2021
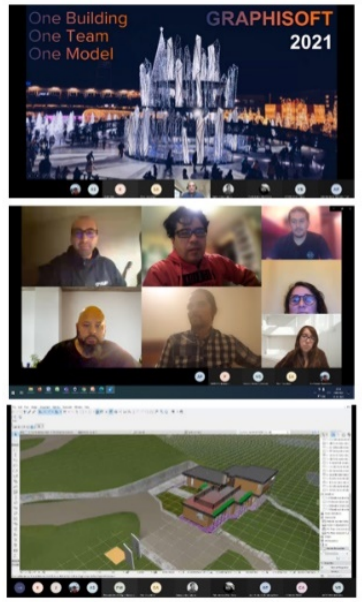

Figura 5 Inicio de Sesión

- Exposición teórica; Ciclo Preguntas y Respuestas;

Demostración de Interfaz del Software. Fuente: Elaboración propia + Víctor Núñez, 2021

\section{INVESTIGACIÓN:}

Los expositores fueron citados a dictar charlas organizadas, secuenciadas y estructuradas, en el que se pidió que se dieran a conocer los siguientes antecedentes relevantes de la marca: 1) Aspectos Teóricos como: Presencia en el mercado regional y nacional, Obras reconocidas a nivel nacional e internacional en los que se hubiera empleado la plataforma BIM, Antecedentes de contacto del representante oficial de la marca en el país, Acceso a ofertas de certificación académica de la marca, Disponibilidad para acceder gratuitamente a versiones de Demostración o Licencias para Estudiantes. 2) Sesión de demostración práctica de aspectos básicos como: Modo de funcionamiento y ubicación de los comandos en la interfaz, para diseño y modelado de elementos básicos tales como muros, losas, puertas y ventanas. Luego componer 
láminas. Imprimir en formato PDF y exportar modelos en diferentes formatos libres, sobre todo IFC.

Tabla 2. Aspectos Prácticos del software (Vectorworks, Allplan, ArchiCAD, Bentley y Revit). Fuente: Elaboración propia, 2021

\begin{tabular}{|c|c|c|c|c|c|c|}
\hline & $\begin{array}{l}\stackrel{0}{\frac{0}{0}} \\
\frac{d}{0} \\
\frac{.0}{0} \\
0\end{array}$ & $\frac{\circ}{\frac{0}{0}}$ & 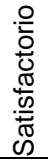 & 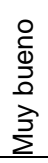 & $\begin{array}{l}\stackrel{\oplus}{\frac{0}{0}} \\
\frac{0}{0} \\
\stackrel{\bigcup}{x} \\
\text { ய }\end{array}$ & 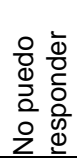 \\
\hline \multicolumn{7}{|l|}{ Facilidad para instalar la versión demo } \\
\hline \multicolumn{7}{|l|}{ Facilidad para acceder a un modelo existente } \\
\hline \multicolumn{7}{|l|}{ Facilidad para explorar la interfaz } \\
\hline \multicolumn{7}{|l|}{$\begin{array}{l}\text { Facilidad para agregar objetos AEC simples } \\
\text { (muro, losa, puerta, ventana, etc.) }\end{array}$} \\
\hline \multicolumn{7}{|l|}{$\begin{array}{l}\text { Facilidad para documentar simple (Una sola } \\
\text { lámina AO con plantas, cortes, tablas básicas), }\end{array}$} \\
\hline \multicolumn{7}{|l|}{$\begin{array}{l}\text { Facilidad para obtener un par de vistas 3D, } \\
\text { insertarlas en la lámina }\end{array}$} \\
\hline $\begin{array}{l}\text { Facilidad para imprimir la lámina a PDF, } \\
\text { exportarla a DWG. }\end{array}$ & & & & & & \\
\hline
\end{tabular}

Se contaba con una presentación comercial de cada Reseller antes de la parte práctica. Al final de cada charla la audiencia especializada respondió una serie de preguntas estructuradas, y orientadas a personas que ya contaban con conocimientos básicos. Se deja constancia que se contestó independientemente si eran usuarios o no de alguno de los softwares expuestos. El cuestionario se generó a través de la herramienta Google Forms y se envió a los correos electrónicos de los participantes. Se realizó de forma obligatoria a los miembros estables de la audiencia participantes del Magíster BIM UTEM. El cuestionario tomaba 10 minutos máximo. Los softwares que se expusieron y los expositores fueron los siguientes. Vectorworks: el workshop y demo se dictaron el día 15 de marzo de 2021, expuso Rubén Hernández F., Arquitecto y Máster Univ. Nacional Pedro Henríquez Ureña, Coordinador Unidad de Arquitectura Avanzada, Miembro de la Sociedad de Arquitectos de la República Dominicana. Se cumplió con el objetivo de demostración y trabajo de manera sincrónica, donde se exploró la interfaz del software y se realizaron modelos y modificaciones simples dentro de una edificación dada. Allplan: el workshop y demo se dictaron el día 17 de marzo de 2021 expusieron José Luis Rodriguez A., Arquitecto, MSc en Urbanismo, Universidad Politécnica de Madrid, experto BIM-GIS. Distribuidor Oficial de Allplan para América Latina, y Sebastián González V., Socio Fundador TB Arquitectos, Máster @ Polis-Maker for the Quality of Life and Sustainable Urban Development, Politécnico di Milano, Micromaster Leadership in Global, The University of Queensland, Arquitecto, Universidad de Talca. Se cumplió con el objetivo de demostración del software. ArchiCAD: el workshop y demo se dictaron el día 18 de marzo de 2021. Charlista Víctor Núñez Bustos, Magíster en Construcción Pontificia Universidad Católica de Chile | Diplomado Arquitectura Sustentable PUC | Arquitecto UDD | Instructor e investigador BIM ArchiCAD y 
Archiframe madera | Consultor Arquitectura Paramétrica. Bentley: el workshop y demo se dictaron el día 25 de marzo de 2021, expuso Mario Perez, director de BIM Nexus, Reseller de Bentley en Chile. Revit: se consideró una excepción ya que se expuso durante el primer semestre del magíster en tecnologías BIM en el módulo de Modelamiento dentro de las clases, por los profesores Danny Lobos y Alejandro Lanfranco.

Finalmente, todas las respuestas fueron procesadas en una sola matriz desde donde se derivaron los análisis mostrados más adelante. Matriz de encuesta realizada por cada software presentado en los Workshop:

Tabla 1. Aspectos Teóricos del software (Vectorworks, Allplan, ArchiCAD, Bentley y Revit). Fuente: Elaboración propia, 2021

\begin{tabular}{|c|c|c|c|c|c|c|}
\hline & $\begin{array}{l}\frac{0}{0} \\
\frac{0}{0} \\
. \frac{10}{0} \\
0 \\
0\end{array}$ & $\begin{array}{l}\frac{0}{\overline{0}} \\
\sum \\
\end{array}$ & $\begin{array}{l}\frac{0}{0} \\
\frac{0}{0} \\
\frac{\pi}{\omega N} \\
\frac{\pi}{\pi} \\
\omega\end{array}$ & 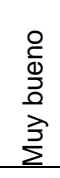 & 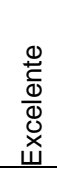 & 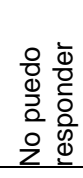 \\
\hline \multicolumn{7}{|l|}{ Facilidad para reconocer Origen del software } \\
\hline \multicolumn{7}{|l|}{$\begin{array}{l}\text { Facilidad para reconocer oficinas que lo usan chile y } \\
\text { el mundo. }\end{array}$} \\
\hline \multicolumn{7}{|l|}{ Facilidad para reconocer proyectos emblemáticos } \\
\hline \multicolumn{7}{|l|}{$\begin{array}{l}\text { Facilidad para reconocer al Reseller chileno y costos } \\
\text { de las licencias. }\end{array}$} \\
\hline
\end{tabular}

Al final había además dos preguntas de respuesta larga para evaluar aspectos generales: ¿Qué aspectos de este software te resultaron más útiles? (Vectorworks, Allplan, ArchiCAD, Bentley y Revit), y ¿Qué aspectos de este software le mejorarías? (Vectorworks, Allplan, ArchiCAD, Bentley y Revit). Respecto de Vectorworks ¿Qué aspectos de este software te resultaron más útiles? Hubo ocho respuestas de texto: "Visualización y fácil accesibilidad a las herramientas, Interfaz muy intuitivo y amable, Lo liviano de los archivos 3D al momento de navegar y dibujar, GIS, El ingreso de datos, Interfaz, No logre trabajar con el software para tener mayor contacto con la interfaz". ¿Qué aspectos de este software mejorarías? Hubo siete respuestas: "Por ahora nada. Disminuir ventanas de información. Resumen de herramientas. Debería ser más amigable para instalar. Bibliotecas. No logré trabajar con el software"

Respecto de Bentley se preguntó ¿Qué aspectos de este software te resultaron más útiles? Hubo siete respuestas: "Herramientas e interfaces con las que cuenta, La interoperabilidad como concepto base, y las posibilidades de administración, coordinación y comunicación entre actores del proyecto. Realmente destacable el potencial expuesto de las herramientas Bentley, en especial "Synchro". Todo. Su Interoperabilidad. Su sistema de compresión de archivos. Sin respuesta". ¿Qué aspectos de este software mejorarías? Seis respuestas: "Interfaz visual. No podría decirlo sin probarlo. El aspecto de la interfaz y herramientas. Su interfaz. Sin respuesta" 


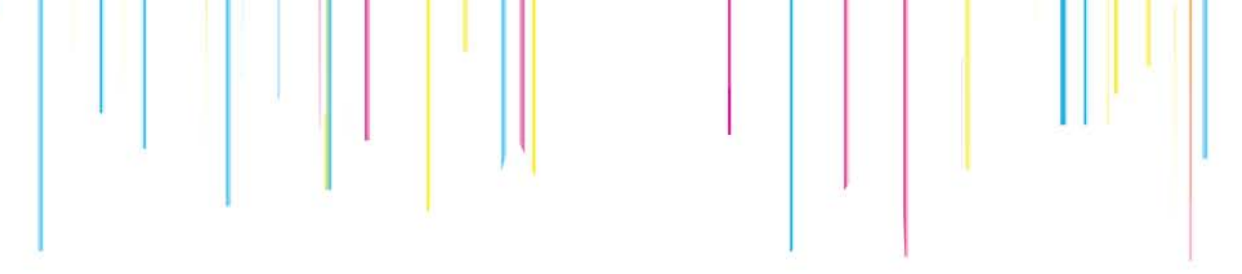

Respecto de Revit: ¿Qué aspectos de este software te resultaron más útiles? Ocho respuestas de texto: "Insertar modelos y familias. La transversalidad para trabajar frente a cualquier tipo de proyecto. Amplia disponibilidad de información y productos. Elementos para modelar superficies complejas. Lo amigable del Software. Vista 3D, cubicaciones e interferencia. Creación de láminas, cubicaciones. Modelado de elementos constructivos". ¿Qué aspectos de este software mejorarías? Ocho respuestas: "Crear un origen UTM. Mejor desempeño del software para modelos estructurales y de infraestructura. La intuitividad de uso, interfaz más amigable. Simplificar los procedimientos para realizar una determinada entidad. Mejoraría la detección de interfaces 4D y 5D. Que tuviera más familias cargadas. Formas curvas, render. Edición de familias"

\section{Análisis:}

A continuación, se muestran los gráficos de análisis de aspectos teóricos de los distintos softwares BIM con presencia en Latinoamérica (Figura 6 a la 9): Facilidad para reconocer Origen del software, Facilidad para reconocer oficinas que lo usan chile y el mundo, Facilidad para reconocer proyectos emblemáticos, Facilidad para reconocer al Reseller chileno y costos de las licencias, Facilidad para acceder a la certificación internacional de la marca.

Se desprende de la Figura 7 que los softwares donde más fácilmente se reconoce las oficinas en Chile y el mundo son Allplan, seguido de Revit y Archicad. y el más difícil de reconocer oficinas en Chile y el mundo es Bentley seguido de Vectorworks. Es notorio que con solo un ejemplo bien mostrado de parte de Allplan, este software está liderando la categoría, seguido de Revit, que sabemos invierte millones de dólares en su publicidad anualmente. 


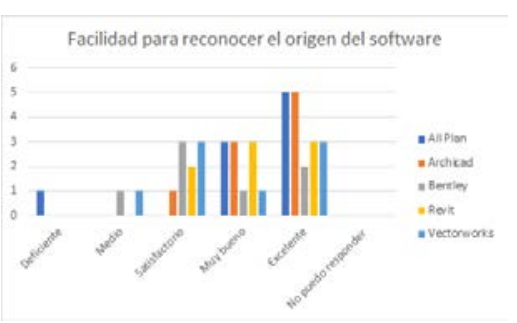

Figura 6. Facilidad para reconocer Origen del software. Fuente: Elaboración propia, 2021.

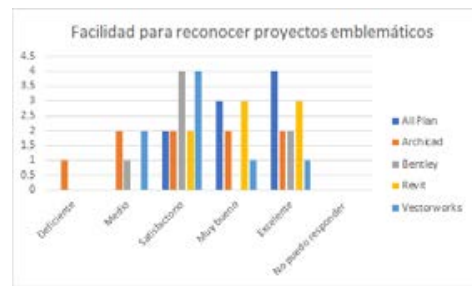

Figura 8. Facilidad para reconocer proyectos emblemáticos. Fuente: Elaboración propia, 2021.

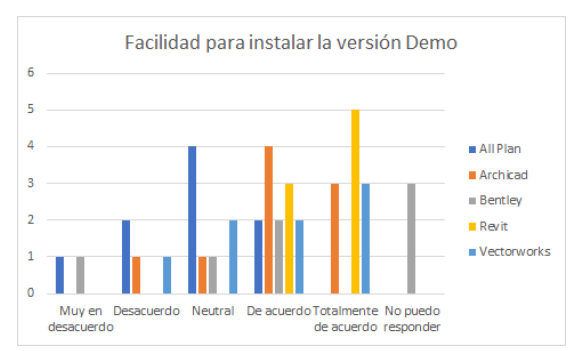

Figura 10. Facilidad para instalar la versión demo. Fuente: Elaboración propia, 2021.

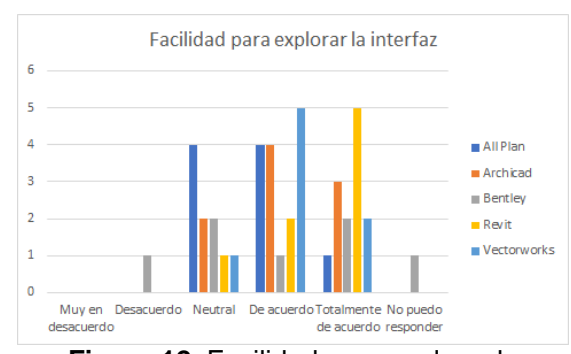

Figura 12. Facilidad para explorar la interfaz. Fuente: Elaboración propia, 2021.

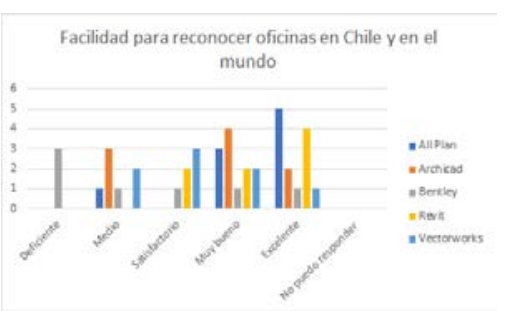

Figura 7. Facilidad para reconocer oficinas que lo usan Chile y el mundo. Fuente: Elaboración propia, 2021.

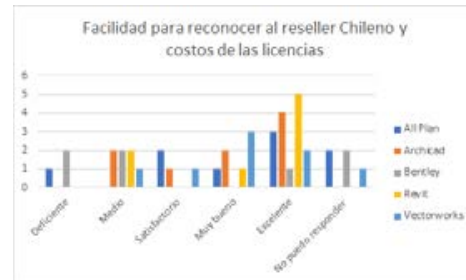

Figura 9. Facilidad para reconocer al Reseller chileno y costos de las licencias. Fuente: Elaboración propia, 2021.

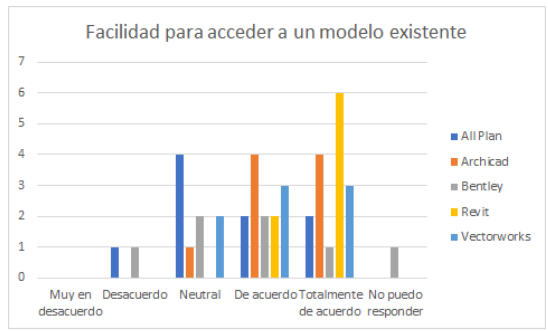

Figura 11. Facilidad para acceder a un modelo existente. Fuente: Elaboración propia, 2021.

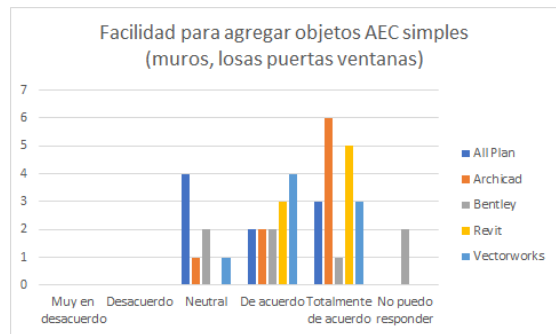

Figura 13. Facilidad para agregar objetos AEC simples (muro, losa, puerta, ventana, etc). Fuente: Elaboración propia, 2021 
A continuación, se muestran los gráficos de análisis de aspectos prácticos de los distintos softwares BIM con presencia en Latinoamérica (Figura 10 a la 17): Facilidad para instalar la versión demo. Facilidad para acceder a un modelo existente. Facilidad para explorar la interfaz. Facilidad para agregar objetos AEC simples (muro, losa, puerta, ventana, etc). Facilidad para documentar simple (Una sola lámina A0 con plantas, cortes, tablas básicas). Facilidad para obtener un par de vistas 3D, insertarlas en la lámina. Facilidad para imprimir la lámina a PDF, exportar a DWG. Facilidad para exportar-importar modelo a IFC.

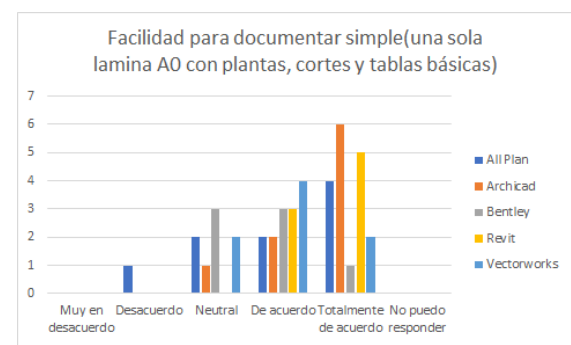

Figura 14. Facilidad para documentar simple (Una sola lámina A0 con plantas, cortes, tablas básicas). Fuente: Elaboración propia, 2021.

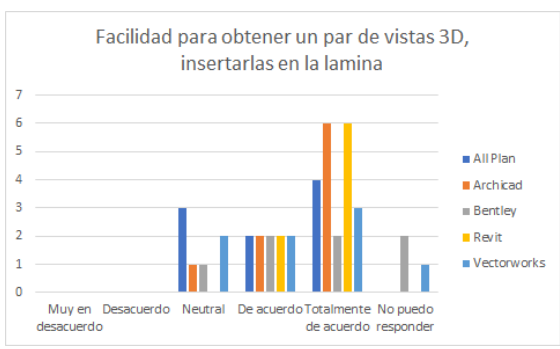

Figura 15. Facilidad para obtener un par de vistas 3D, insertarlas en la lámina. Fuente: Elaboración propia, 2021.

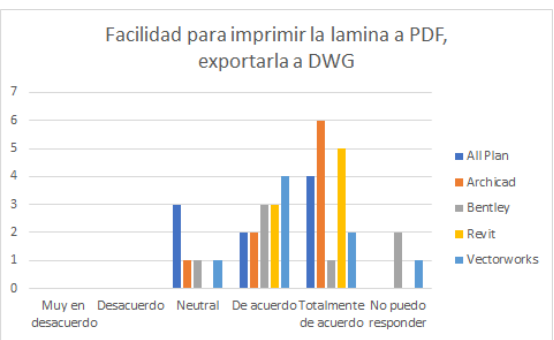

Figura 16. Facilidad para imprimir la lámina a PDF, exportar a DWG. Fuente: Elaboración propia, 2021.

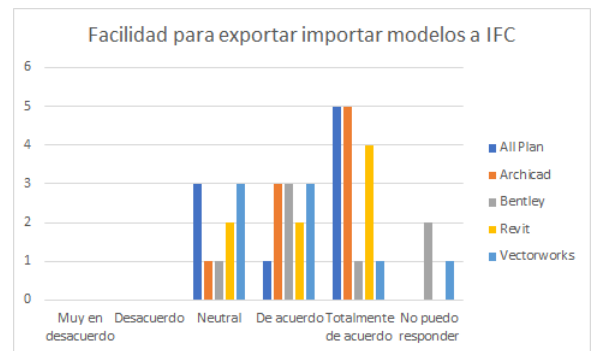

Figura 17. Facilidad para exportarimportar modelo a IFC. Fuente: Elaboración propia, 2021.

\subsection{Resultados parte práctica}

Respecto de la pregunta "Facilidad para instalar la versión demo", se ve que los softwares más fáciles de instalar eran Revit, Archicad, seguido de Vectorworks, y los más complejos eran Bentley y Allplan. Respecto de la pregunta "Facilidad para acceder a un modelo existente", se ve que los 
softwares más fáciles para acceder a un modelo existente eran Revit, Archicad, seguido de Vectorworks, y los más complejos eran Bentley y Allplan. Respecto de la pregunta "Facilidad para explorar la interfaz", se ve que los softwares más fáciles para explorar la interfaz eran Revit, Vectorworks, seguido de Archicad y Allplan, y el más complejo era Bentley. Respecto de la pregunta "Facilidad para agregar objetos AEC simples (muro, losa, puerta, ventana, etc)", se ve que los softwares más fáciles para agregar objetos eran Archicad, Revit, seguido de Vectorworks, y los más complejos eran Bentley y Allplan. Respecto de la pregunta "Facilidad para documentar simple (Una sola lámina AO con plantas, cortes, tablas básicas)", se ve que los softwares más fáciles para documentar eran Archicad, Revit, seguido de Vectorworks y Allplan, y el más complejo era Bentley.

Respecto de la pregunta "Facilidad para obtener un par de vistas 3D, insertarlas en la lámina", se ve que los softwares más fáciles para obtener un par de vistas 3D eran Revit, Archicad, seguido de Allplan, y los más complejos eran Vectorworks y Bentley. Respecto de la pregunta "Facilidad para imprimir la lámina a PDF, exportar a DWG", se ve que los softwares más fáciles para imprimir la lámina a PDF Archicad, Revit, seguido de Allplan, y los más complejos eran Vectorworks y Bentley. Respecto de la pregunta "Facilidad para exportar-importar modelo a IFC", se ve que los softwares más fáciles para exportar-importar modelo a IFC eran Archicad, Allplan, seguido de Revit, y los más complejos eran Vectorworks y Bentley.

\section{CONCLUSIONES GENERALES}

Se observó que no todas las empresas cumplieron las expectativas sobre el temario propuesto, algunas no abordaron todos los temas requeridos, ejemplo origen software, licencias en el país y presencia comercial, era a veces confusa. Las respuestas de los asistentes muestran que todos los softwares tenían un desempeño similar en términos generales (modelamiento, precios), pero sin embargo mostraron diferencias significativas en aspectos teóricos tales como ejemplos conocidos, certificación, etc.

\section{DISCUSIÓN}

La mayoría de las marcas de software BIM, están presentes en el mercado Latinoamericano, solo faltó Digital Project, software TEKLA no estuvo disponible en esta versión, pero sí será incluido en el futuro. Se ve la necesidad de avanzar en la difusión de los desempeños de las distintas marcas en la industria AEC en Latinoamérica, es evidente el sesgo comercial y marketing de algunas pocas marcas. Temas de precios no son la barrera para elegir una $u$ otra. No todos los representantes tenían el mismo enfoque ni estrategia de exposición. El nivel de experiencia individual influye fuertemente en la valoración del software. El perfil del usuario y el tipo de proyecto van a determinar el software a utilizar. El disímil currículum de los expositores radica en amplias posibilidades de conocer diversas experiencias, pero también en 
